\title{
Management of insomnia: update and new approaches
}

\author{
This article was published in the following Dove Press journal: \\ Nature and Science of Sleep \\ 23 July 2010 \\ Number of times this article has been viewed
}

\section{Thomas Unbehaun \\ Kai Spiegelhalder \\ Verena Hirscher \\ Dieter Riemann \\ Department of Psychiatry and Psychotherapy, University of Freiburg Medical Center, Freiburg, Germany}

Correspondence:Thomas Unbehaun Department of Psychiatry and Psychotherapy, University of Freiburg Medical Center, Hauptstraße 5, 79I04 Freiburg, Germany

$\mathrm{Tel}+49$ 76| 270-698 I

Fax +4976I 2706619

Email thomas.unbehaun@uniklinik-freiburg.de
Abstract: Insomnia is the most prevalent sleep disorder worldwide. A number of studies evaluated the efficacy of pharmacological and nonpharmacological treatment approaches. To obtain long-term effects in the management of chronic insomnia, cognitive-behavioral therapy for insomnia (CBT-I) is the treatment of first choice, encompassing education about sleep and sleep hygiene, sleep restriction, stimulus control, relaxation techniques, and cognitive strategies to combat nocturnal ruminations. Short-term effects can easily be achieved by the administration of hypnotic drugs. Gaining access to all types of treatment can still be considered a problem, especially CBT-I seems to be available only at specialized centers but not in general health care. New approaches to treatment delivery seem to be necessary to provide adequate care for patients who may seek help or have not entered the health care system yet. Internet-based treatment options and stepped-care models might be feasible options for the future. Otherwise, the direct and indirect costs associated with insomnia might further increase for our societies, in addition to the personal impact on aspects of quality of life and impaired daytime functioning for each individual with insomnia. Besides, well-established psychological and pharmacological treatment options, alternative treatments like acupuncture might constitute new nonpharmacological possibilities. Randomized controlled studies are needed to evaluate the efficacy of this and other new approaches to treat insomnia.

Keywords: treatment, access, pharmacological, CBT-I

\section{Introduction}

Insomnia, in terms of a diagnostic entity, is defined as having difficulties to fall asleep or to maintain sleep during the night, or early morning wakening and consequently experiencing impairments in daytime functioning for at least 4 weeks. Consequently, patients worry and ruminate about their symptoms at night and are concerned about the expected negative consequences during daytime. These symptoms are very common, frequently occurring comorbid with almost any mental or organic disorder, and also as an independent sleep disorder (primary insomnia [PI]; seeTable 1). The Diagnostic and Statistical Manual of Mental Disorders, Fourth Edition, Text Revision (DSM-IV-TR) of the American Psychiatric Association differentiates three types of insomnia: PI, insomnia related to a medical or mental disease, and insomnia related to the intake or abuse/dependency from substances. ${ }^{1}$ If the symptoms persist for at least 6 months, chronic insomnia is being diagnosed.

Comparing both diagnostic systems, the International Classification of Diseases 10 (ICD-10) and the DSM-IV-TR, a few differences occur in the definition of insomnia. The ICD-10 considers early final awakenings as one of the symptoms, whereas 
Table I Diagnostic criteria for nonorganic insomnia according to ICD- $10^{87}$ and DSM-IV-TR

ICD-I0

Difficulty falling asleep, maintaining sleep, early

final wakening, or nonrestorative sleep

The sleep disorder occurs at least 3 nights per

week and retains for at least I month

The unsatisfactory sleep duration or sleep quality

causes a significant suffering or impairments in

daytime functioning

The sleep problems cannot be explained by any organic causes, eg, neurological or other somatic diseases or impairments or use of psychotropic substances or medication

DSM-IV-TR

The predominant symptom is difficulty initiating

or maintaining sleep, or nonrestorative sleep

for at least I month

The sleep disturbance (or associated daytime

fatigue) causes clinically significant distress or

impairment in social, occupational, or other

important areas of functioning

The sleep disturbance does not occur exclusively during the course of narcolepsy, breathing-related sleep disorder, circadian rhythm sleep disorder, or a parasomnia

The disturbance does not occur exclusively during the course of another mental disorder (eg, major depressive disorder, generalized anxiety disorder, and a delirium)

The disturbance is not due to the direct

physiological effects of a substance (eg, drug abuse and medication) or a general medical condition

Abbreviations: DSM-IV-TR, Diagnostic and Statistical Manual of Mental Disorders, Fourth Edition, Text Revision; ICD-I0, the International Classification of Diseases I0.

DSM-IV-TR does not. The ICD-10 also specifies the frequency (at least 3 nights per week) for the occurrence of symptoms, in addition to the duration of at least 1 month, which is the same in both diagnostic systems. Both address the consequences of sleep disturbances for daytime functioning. Being more specific concerning organic causes, the DSM-IV-TR is also ruling out any mental disorder in order to diagnose a nonorganic insomnia. Both address the use of psychotropic substances or medication as an exclusion criterion for the diagnosis as well. However, commonly used by sleep specialists and in the field of clinical sleep research, the International Classification of Sleep Disorders (ICSD-2) ${ }^{2}$ specifies 11 subtypes of insomnia (see Table 2).

In a recent epidemiological study conducted by Ohayon in five European countries (France, United Kingdom, Germany, Italy, and Spain), a representative sample of 22,740 noninstitutionalized individuals, aged 15-101, were interviewed by phone. ${ }^{3}$ In this study, $16.1 \%$ reported nocturnal awakenings with difficulties to resume sleep. Out of 8,937 individuals from New York, California, and Texas (aged 18 years and older), 34.2\% reported having
Table 2 ICSD-2 subtypes of insomnia

Acute (or adjustment) insomnia

Psychophysiological insomnia

Paradoxical insomnia

Idiopathic insomnia

Insomnia because of a mental disorder

Insomnia because of inadequate sleep hygiene

Behavioral insomnia of childhood

Substance-induced insomnia

Insomnia due to medical condition

Insomnia not due to substance or known physiologic condition -

unspecified (nonorganic insomnia, not otherwise specified)

Physiologic (organic) insomnia - unspecified

Abbreviation: ICSD-2, International Classification of Sleep Disorders-2.

more than three nocturnal awakenings per week for more than 1 month, including $19.3 \%$ with daytime impairments. Almost 9\% reported difficulties initiating sleep ${ }^{4}$ and more than $90 \%$ suffered from nocturnal awakenings for more than 6 months. Once awake, approximately $50 \%$ of them reported difficulties to resume sleep. According to a study conducted by LeBlanc et $\mathrm{al}^{5} 30.7 \%$ of the population had insomnia symptoms and $7.4 \%$ had an insomnia syndrome within a year, among which $28.8 \%$ and $3.9 \%$, respectively, had newly developed insomnia during this time span. These incidence rates illustrate the fact that insomnia affects a large part of the population.

Chronic insomnia is associated with reduced quality of life and impaired psychosocial functioning, ${ }^{6}$ including increased fatigue, cognitive impairments, negative mood swings, physical discomfort, and an increased utilization of medical services. Suffering from chronic insomnia also increases the risk for developing a psychiatric disorder, such as major depression and substance abuse/dependency. ${ }^{7-9}$ Furthermore, insomnia also has an impact on medical conditions. Vgontzas et al showed an association between short sleep durations ( $<5$ hours) and an increased risk for hypertension. ${ }^{10,11}$ Insomnia is also known for affecting the regulation of blood glucose and the functions of the immune system. ${ }^{12-14}$ This highlights the need to focus on the treatment of insomnia in order to prevent medical and mental sequel disorders. ${ }^{15}$

According to current etiological theories, insomnia is probably initiated and maintained by hyperarousal on a cognitive, emotional, motor, and physiological level. ${ }^{16,17}$ Many patients are unable to "turn off" their thoughts when trying to sleep, ruminating about sometimes even mundane daytime events and experiencing negatively toned thoughts or fears about the detrimental effects of not being able to sleep. Insomnia patients frequently "misperceive" their sleep duration and quality, overestimating the time awake at night 
or underestimating the duration of their sleep compared with polysomnographic recordings of their sleep. Physiological hyperarousal has been measured with various autonomous indicators, eg, blood pressure or heart rate. ${ }^{18-20}$ Differences between good and poor sleepers have also been found for cortisol secretion at night and an increase of interleukin- $6,{ }^{21,13}$ as well as an increased beta power in the power spectral analysis of the sleep EEGs. ${ }^{22,23}$ The last one is regarded as a neurophysiological correlate of cognitive nocturnal hyperarousal subjectively experienced as being unable to "shut off" thoughts. Regarding their sleep - wake behavior, patients with PI intuitively develop maladaptive patterns assuming to improve and promote their sleep, such as staying in bed for a longer period of time, going to bed earlier, daytime naps, reading, working, or watching TV in bed. ${ }^{24}$ Contrary to common sense intuition, all of these activities negatively interfere with sleeping.

Genetic factors seem to affect the emergence and perpetuation of insomnia. Patients with PI have more first-degree relatives with PI than patients with secondary insomnia. ${ }^{25}$ In addition, women are more often affected than men with a risk ratio of 1.41 reported by Zhang and Wing in a meta-analysis of 31 papers and 718,828 female vs 546,187 male participants. ${ }^{26}$

\section{Daytime impairments}

As part of the symptomatology of insomnia, patients experience impairments during the day after nights of poor sleep. Insomnia has been proven to exert a negative impact on health-related quality of life, ${ }^{27}$ limiting domains like energy and vitality, and also aspects of social, physical, and mental functioning. These impairments are linearly associated with the severity of insomnia. Various research groups have tried to measure and evaluate the impaired functioning with the help of diverse psychometric tests to detail corroborating evidence, eg, performance tests on vigilance, working memory, motor control, or other cognitive domains. ${ }^{28}$ A recently published meta-analysis by Shekleton et $\mathrm{al}^{29}$ evaluated the few studies that can be found investigating neurobehavioral performance deficits in insomnia patients. The results are inconsistent and findings are conflicting. According to the authors, deficits seem to be only subtle. Working memory tasks and attention tasks appear to reveal performance deficits. The inability to prove distinct daytime impairments is assumed to be due to heterogeneous patient populations, differing test protocols and conditions, and also unsuitable cognitive tasks in the primary literature. Another reason might be the assumed hyperarousal in insomnia patients, which might result in increased compensatory effort.

\section{Cost-effectiveness and acceptance of therapy}

Martin and colleagues ${ }^{30}$ searched MEDLINE for available literature and published a review of cost-effectiveness analyses in the treatment of insomnia in 2004. The authors conclude that insomnia management holds promises as a cost-effective intervention; however, accurate measurements of direct and indirect costs are required and estimations of costs varied widely between the original papers.

Daley et $\mathrm{al}^{31}$ recently evaluated the annual economic costs caused by insomnia for the population of the province of Quebec, Canada. Data of 948 adults and data from the Quebec government administered health insurance board were analyzed to estimate direct and indirect costs - in sum, \$6.6 billion per year: \$191.2 million for insomnia-motivated health care consultations, $\$ 36.6$ million for transportation to the consultations, $\$ 16.5$ million for prescribed drugs, \$1.8 million for over-the-counter products, \$339.8 million for alcohol (as a sleep aid), an estimated amount of $\$ 970.6$ million for insomnia-related absenteeism, and $\$ 5.0$ billion for insomnia-related productivity losses. The reported direct and indirect annual costs per person for individuals with full-blown insomnia syndrome were $\$ 5,010$, for individuals with insomnia symptoms were $\$ 1,431$, and for individuals with good sleep were $\$ 421$ (all sums in Canadian dollars).

An American study ${ }^{32}$ evaluated the economic impact of insomnia and compared the prevalence and costs of comorbidities in employees with and without insomnia, using data from the Human Capital Management Services Research Reference Database from January 2001 to September 2007. The criteria for the insomnia group $(n=17,230)$ were a history of receiving medication to treat insomnia or a physician's diagnosis of insomnia. The control group contained 281,958 employees having other disorders. For employees with insomnia, annual mean incremental costs were US\$2,053 greater in total: \$751 for medical services, \$735 for medication, \$208 for sick leave, $\$ 179$ for short-term disability, $\$ 10$ for long-term disability, and \$170 for workers' compensation. Compared to those without insomnia, they missed 3.10 workdays more each year. The occurrence of insomnia increases the prevalence of comorbid conditions, which are more costly and lead to a greater utilization of health services.

Of note, the above reported data is only for those using the health care system. Costs of untreated insomnia might be 
much higher. However, it is difficult to estimate all indirect costs related to this issue.

Patients' and significant others' (mostly spouses of patients) acceptance of psychological or pharmacological treatment for insomnia has been studied by Morin et $\mathrm{al}^{33}$ in 71 older adults (mean age, 66.5 years). Thirty-nine of the subjects were seeking treatment for chronic insomnia presenting persistent difficulties initiating or maintaining sleep (mean duration of symptoms, 13.1 years). All subjects were provided with a brief description of a psychological intervention (self-management program trying to change poor sleep habits, regulating sleep schedules, altering dysfunctional thought about sleeplessness, and impact on daytime functioning) and a pharmacological intervention (new hypnotic drug reducing physiological and cognitive arousal) - both being equally effective in treatment. All subjects were asked to rate their opinion about either treatment option on a 100 -mm visual analog scale for each of the 9 items. A higher score indicated a higher acceptance or willingness. Insomnia patients of both sexes, as well as the control subjects, viewed behavioral treatment as more acceptable than pharmacological for themselves (76\% vs $31 \%$ ) and others (74\% vs 39\%), being more suitable for both difficulties: initiating (69\% vs 45\%) and maintaining sleep (68\% vs 45\%). Behavioral treatment was also expected to be more effective on a long-term basis ( $70 \%$ vs $25 \%$ ), producing less side effects (36\% vs 86\%) and more benefits for daytime functioning (69\% vs 31\%).

According to the above-mentioned aspects of treatment for insomnia, a closer look on the currently available treatment options is necessary, and new emerging strategies will be discussed critically.

\section{Treatment options for PI}

Many treatment studies in the field of insomnia are based on DSM-IV criteria for PI or somewhat modified criteria specifying sleep onset problems or sleep maintenance disturbances and duration of insomnia. Therefore, the following studies are mainly representatives for therapeutic interventions dealing with PI. First, the interventions can be differentiated into pharmacological and nonpharmacological, mostly cognitive-behavioral treatment strategies for insomnia (CBT-I). Combined approaches, including medication and psychotherapy, have also been tested for superiority compared with single approaches. Research indicates that cognitive-behavioral therapy alone is superior to the intake of psychotropic substances in the long run. ${ }^{34}$
The National Institutes of Health (NIH) reported results from the State-of-the-Science Conference in 2005, criticizing that there are mainly randomized controlled drug studies regarding the short-term effectiveness (3-4 weeks of administration) of hypnotic drugs. Only two long-term studies have been published - a striking finding - given the fact that in clinical practice, sleep medications are prescribed for long periods of time very frequently.

\section{Pharmacological strategies}

What different pharmacological strategies are available? The sedating drugs can be differentiated into older benzodiazepines (BZs) and more recently developed so-called Z-drugs (zolpidem, zopiclone, zaleplon, and eszopiclone), sedative antidepressants, low-dose atypical neuroleptics, antihistamines, melatonin, and herbal (= phytotherapeutic)

Table 3 Hypnotics and other psychotropic drugs used for the treatment of insomnia

\begin{tabular}{|c|c|}
\hline \multirow[t]{4}{*}{ Z-drugs } & Zolpidem \\
\hline & Zopiclone \\
\hline & Eszopiclone \\
\hline & Zaleplon \\
\hline \multirow[t]{9}{*}{ Benzodiazepines } & Triazolam \\
\hline & Lormetazepam \\
\hline & Brotizolam \\
\hline & Diazepam \\
\hline & Alprazolam \\
\hline & Temazepam \\
\hline & Loprazolam \\
\hline & Nitrazepam \\
\hline & Flunitrazepam \\
\hline \multirow[t]{6}{*}{ Sedating antidepressants } & Mirtazapine \\
\hline & Trimipramine \\
\hline & Doxepin \\
\hline & Amitriptyline \\
\hline & Mianserin \\
\hline & Trazodone \\
\hline \multirow[t]{7}{*}{ Antipsychotics } & Melperone \\
\hline & Pipamperone \\
\hline & Promethazine \\
\hline & Thioridazine \\
\hline & Chlorprothixene \\
\hline & Levomepromazine \\
\hline & Promazine \\
\hline \multirow[t]{2}{*}{ Antihistamines } & Diphenhydramine \\
\hline & Doxylamine \\
\hline Alcohol derivative & Chloral hydrate \\
\hline \multirow[t]{6}{*}{ Herbal drugs } & Valerian \\
\hline & Chamomile \\
\hline & Balm \\
\hline & Lavender \\
\hline & Hops \\
\hline & Passion-flower \\
\hline
\end{tabular}


drugs. A selection of most commonly prescribed hypnotics is shown in Table 3.

So far, there are five published meta-analyses evaluating the efficiency of BZ receptor agonists for a short-term treatment of 4 weeks at most. Most of the analyzed data were derived from sleep diaries, where patients can record their bedtimes, experienced sleep quality, number of nocturnal awakenings, or sleep onset latency the next morning. So far, sleep diaries are widely used as an economical diagnostic instrument.

In a first meta-analysis ${ }^{35}$ of 22 studies with overall 1,894 middle-aged patients (approximately $60 \%$ were women), the effect sizes of classic BZ drugs and zolpidem were analyzed. In comparison to placebo, the hypnotic drugs had effect sizes of 0.56 for sleep onset latency, 0.71 for the number of nocturnal awakenings, and 0.65 for the total sleep time. These effect sizes can be interpreted as medium-sized therapeutic effects for the examined substances.

In a second meta-analysis by Holbrook and colleagues, ${ }^{36}$ 45 studies with 2,672 patients were included. Forty-seven percent of the study population were women. The mean age ranged from 29 to 82 years, with 15 studies that included patients over 65 years of age. In these studies, the mean duration of drug intake was 7 days. Treatment differences were not reported in effect sizes, instead the authors used confidence intervals. In a polysomnographic comparison to placebo, no reduction of sleep onset latency was found for BZ receptor agonists. However, total sleep time was increased by more than 60 minutes. The analysis of the sleep diaries showed a significant reduction in subjectively reported sleep onset latency of 14 minutes.

Duendar et $\mathrm{al}^{37}$ evaluated the newer hypnotics (Z-drugs: zolpidem, zopiclone, zaleplon) in comparison to classic BZ hypnotics, not only in terms of the short-term efficacy but also concerning the side effects and economic aspects. Twenty-four studies with 3,909 participants were included. The main result was that only minor differences were found between the drug classes. Patients with zolpidem had a lowered probability to develop a rebound insomnia, as well as a reduced sleep onset latency compared with patients with zopiclone. In general, both substances did not show any major differences to classic BZ hypnotics. Therefore, the authors critically concluded that newer Z-drugs and older BZ drugs are equally effective, with the newer hypnotics being more expansive for health care systems.

Glass et $\mathrm{al}^{38}$ analyzed the effectiveness of sedating hypnotics on patients, who were at least 60 years old. Including 24 studies with 2,417 participants, the meta-analysis compared zopiclone, zolpidem, BZ, like triazolam and temazepam, and the antihistamine diphenhydramine. The analysis showed a significant effect of all substances on subjectively reported sleep quality, though with only a small effect size of 0.14 . The outcome on other sleep parameters was also significant, though again small in effect size. Furthermore, the study presented a heightened risk of undesirable cognitive and psychomotor side effects. Compared with placebo, the odds ratios were 4.8 for adverse cognitive events, 3.8 for daytime fatigue, and 2.6 for adverse psychomotor events. In summary, the small benefit in the use of sedating hypnotics for patients older than 60 years does not seem to redeem the occurring side effects and risks.

The latest meta-analysis included 52 studies on BZ hypnotics and 48 studies on Z-drugs with primarily nonelderly subjects and both genders, but once again no between-drug differences were found to be significant. ${ }^{39}$ The polysomnographically measured sleep onset latency was significantly reduced in the treatment with BZ (10 minutes vs placebo), as well as in the use of Z-drugs (12.8 minutes vs placebo). In the sleep diaries, the sleep onset latency was reduced by 19.6 concerning BZ compared with placebo and 17.0 minutes by Z-substances.

In summary, the meta-analyses of Nowell et $\mathrm{al}^{35}$ and Holbrook et $\mathrm{al}^{36}$ suggest a significant clinical effect of BZ receptor agonists with medium effect sizes for a short-term use of 3-4 weeks. Comparative meta-analyses do not show an increased efficacy of the Z-drugs compared with older BZ. The analysis in elderly patients ( $>60$ years) concluded that the increased side effects of the therapy outweigh the clinical benefit in these patients.

The problem of drug tolerance and dependence is a major concern in the long-term treatment of insomnia with hypnotics. Therefore, the classical BZ and the Z-drugs are only approved to be used on a short-term basis with a maximum of 4- or 6-week treatment duration in most countries. The longterm usage of $\mathrm{BZ}$ and $\mathrm{BZ}$ receptor agonists (BZRA) is usually not recommended. Especially for patients with a known risk for abuse/dependency (eg, a prior history of such) to develop an addictive disorder, BZs are strictly contraindicated because of an explicitly increased risk of dependence.

Until now, there are three published placebo-controlled double-blinded studies conducted by Krystal and colleagues on the long-term efficacy (6 months) of eszopiclone, ${ }^{40}$ zolpidem, ${ }^{41}$ and zaleplon. ${ }^{42}$ Taking eszopiclone, the active dextrorotatory stereoisomer of zopiclone, patients with PI, aged 18-65 years, experienced a significantly shortened sleep onset latency, less nocturnal time awake, and an increased 
total sleep time. These effects were relatively stable over 6 months. Moreover, no signs of severe side effects were reported during the 6-month treatment period with eszopiclone. In the second study of Krystal et al zolpidem was compared with placebo and tested in 1,018 patients suffering from PI. The treatment lasted for 6 months. Significant effects were found for zolpidem on the sleep onset latency, number of nocturnal awakenings, total sleep time, and daytime fatigue, as measured by subjective data from questionnaires filled out in the next morning. Similar to the eszopiclone study, relatively stable effects were reported for the 6-month period without severe side effects. Furthermore, a positive effect on daytime work performance was found..$^{43}$ Ancoli-Israel et $\mathrm{al}^{42}$ conducted a 1-year open-label extension phase of 2 doubleblind randomized trials on zaleplon in elderly patients who self-administered the drug ( 5 or $10 \mathrm{mg}$ ) during night for a period of 6 months. The authors report a maintained significant improvement on sleep onset latency, total sleep time, and number of nocturnal awakenings without the effect of any rebound insomnia in case of discontinuation.

In recent years, pharmaceutical companies have developed and introduced new forms of medicines to the market, trying to facilitate the intake. Two examples are Zolpimist ${ }^{\circledR}$ (NoraDel Pharma, Bridgewater, NJ), an oral spray, and Edluar $^{\circledR}$ (Meela Pharmaceuticals, Somerset, NJ), a sublingual tablet - both containing the active ingredient zolpidem.

Low-dose sedative antidepressants are now widely used in clinical practice for the treatment of insomnia in Europe and the United States. ${ }^{44}$ However, only one meta-analysis has been published on this topic, ${ }^{39}$ including eight original studies. Compared with BZ receptor agonists, sedative antidepressants showed a marginal reduced efficacy. According to the original reports, trimipramine, doxepin, and trazodone lead to a significant improvement in insomnia symptoms. ${ }^{45-48}$ However, further studies on the long-term efficacy of sedative antidepressants are needed. In clinical practice, mirtazapine is often prescribed by physicians for treatment. However, no randomized placebo-controlled studies have been published yet investigating the effects of the drug on insomnia per se.

A major issue in the treatment with sedative antidepressants is the question of undesirable side effects, especially in the treatment of elderly patients. Caution seems to be appropriate due to the fact that most antidepressants have a high potential for cardiovascular, urogenital, or gastrointestinal side effects. Furthermore, there are no studies up to now that investigated weight gain in insomnia patients treated with antidepressants.
Until now, there is a total lack of placebo-controlled studies on the effect of antipsychotics on insomnia. Because of the sleep enhancing and sedative effect of many antipsychotics, ${ }^{49}$ such as risperidone, haloperidol, quetiapine, or olanzapine, they are widely used for treatment in older patients. However, expert group reviews and meta-analyses ${ }^{50}$ keep warning of an uncritical and uncontrolled prescription for elderly dementia patients due to a significantly increased mortality rate ${ }^{51}$ caused by overdosing.

\section{Alternative strategies}

Melatonin can be purchased in the United States without a prescription as a so-called nutrition supplement, mostly used for the treatment of jet-lag-associated or shift work-related sleep disorders. Meta-analyses did not find any efficacy in the treatment of PI for younger patients. ${ }^{52,53}$ However, a slow release formulation of melatonin (Circadin ${ }^{\circledR}$; Lundbeck, Copenhagen, The Netherlands) has been approved by the European Medical Agency (EMEA) ${ }^{54}$ for the treatment of insomnia patients who are older than 55 years. Melatonin is best used in the treatment of circadian rhythm sleep disorders, such as the delayed sleep phase syndrome (DSPS), as shown by Buscemi and colleagues. ${ }^{52}$

Ramelteon, a melatonin receptor agonist binding at $M_{1}$ and $\mathrm{M}_{2}$ receptors, is the first among a new group of sleep drugs, which has been approved by the US Food and Drug Administration (FDA) in 2005 for the treatment of problems concerning sleep initiation and is also being used for the treatment of DSPS. Unlike BZ/BZ receptor agonists, no side effects, such as dependence or rebound insomnia, have been observed. Due to a low clinical efficiency, ramelteon has not been approved by the EMEA in 2008 and, therefore, has not been introduced to the European market.

A large amount of herbal substances are used worldwide in the treatment of insomnia symptoms, which are mainly used as self-medication. There are three meta-analysis for the use of valerian, concluding that data in support of the effectiveness of this herbal medication is inconclusive. ${ }^{55-57}$ For the other herbal drugs, antihistamines, and alcohol derivates, an evidence-based proof of effectiveness is still missing.

Regarding the treatment of insomnia with acupuncture, in the last few years, some reviews and meta-analyses have been written based on English and Chinese literature. ${ }^{58,59}$ After analyzing 20 randomized controlled trials (RCTs) in detail, Yeung and colleagues concluded that traditional needle acupuncture (TNA) was significantly more effective than BZ having a proportion of subjects who had at least some improvement in their sleep problem after treatment: for TNA, $73 \%-100 \%$, with a mean of 
$91 \%$; for BZ $45 \%-95 \%$, with a mean of $75 \%$. However, limiting factors in these studies are the methodological shortcomings, such as problems with randomization, blinding issues, imprecise diagnostic procedure, and insufficient safety data resulting in mostly poor-quality research designs. Therefore, the superior efficacy of needle acupuncture over other treatments could not be determined. Accordingly, the authors concluded that largescale, placebo-controlled double-blinded trials are necessary to analyze the impact of acupuncture on insomnia. Huang et al also reported that $93 \%$ of 30 analyzed studies showed positive treatment effects of acupuncture on sleep. However, these authors also criticized the limited quality of the original studies due to heterogeneous acupuncture techniques and acupoints, as well as the problem of sham (or placebo treatment) controls. Lee and colleagues reviewed 18 databases for papers dealing with auricular acupuncture and came to the same conclusions as mentioned above. The technique seems to be effective, but in order to confirm the results, better designed trials are necessary. ${ }^{60}$ This raises the questions: Is it really possible to conduct high-quality double-blinded trials with acupuncture and sham controls? And does it mean a "blinded" investigator needs to be trained in a false technique?

\section{Behavioral treatment for insomnia}

This section is divided into two parts. In the first part, we have a close look at the psychological techniques that are successfully used to treat PI. In the second, the results of 5 meta-analyses concerning their effectiveness will be reported.

The main elements of cognitive psychological therapy of insomnia are psychoeducation, stimulus control, sleep restriction, relaxation therapies, and cognitive techniques. At the beginning of the therapy, providing information and presenting a work model of sleep, eg, the two process model by Borbély, ${ }^{61}$ helps many patients to get a better understanding of their disease and is the first step to develop effective coping strategies. Borbély postulates the interaction of the two processes: the sleep-independent circadian process $(\mathrm{C})$ and the sleep-dependent process (S). The model helps to explain why good- and poor-quality nights keep alternating and how cognitive-behavioral interventions can influence sleep quality and the experience of deep vs nonrestorative sleep.

Giving instructions about adequate sleep hygiene is very important, as the rules of sleep hygiene need to be strictly adhered in order to lead to positive effects. The common sleep hygiene rules are given in Table 4 (based on a list published by Hauri ${ }^{24}$ and further developments).
Table 4 Sleep hygiene instructions

I. Keep the bedroom comfortable and free from light and noise

2. The bedroom should have a comfortable room temperature at night

3. Avoid alcohol in the evening, as it causes micro withdrawal symptoms at night

4. Avoid drinking caffeine products after lunch

5. Avoid nicotine as it causes sleep disturbances

6. Reduce liquid intake $2-3 \mathrm{~h}$ before bedtime, so you don't have to go to the bathroom at night

7. Keep the clock out of sight to avoid (activating) frustration at night when you look at it

8. Do not go to bed hungry and eat regular meals, not too heavy or greasy in the evening

9. Do not TRY to fall asleep

10. Use the bed only for sleeping and sexual activity but NOT for reading, drinking, smoking, or watching TV

II. Go to bed only, when you are tired

12. If you are not sleeping after 10-15 min, get up and leave the bedroom. Come back only if you feel tired

13. If you cannot fall asleep for another $10 \mathrm{~min}$, repeat the last point

14. Get up the same time each day to set your "biological clock," regardless of weekends

15. Avoid daytime naps

16. Sleep only as much as you need in order to feel refreshed the next day

17. Exercise relaxation techniques regularly during the day to be able to use them

18. Do not take your problems to bed

Regarding the efficacy of sleep hygiene recommendations in the treatment of insomnia, Morin et $\mathrm{al}^{62}$ conclude in their review that sleep hygiene education is a necessary part of therapy but not a sufficient treatment approach. Stepanski and $\mathrm{Wyatt}^{63}$ present an overview of different sleep hygiene recommendations given by various authors and emphasize the fact that there is quite some variance in these instructions. ${ }^{63}$

An interesting interrelation has been reported by Adan et $\mathrm{al}^{64}$ concerning sleep beliefs of psychology students and the influence of the circadian type and gender. The evening-type students did have more false beliefs (which indicates a risk for wrong sleep hygiene practice and sleep disorders) than morning-type students. Men had more false beliefs than women on the Sleep Beliefs Scale (SBS) -8 out of 20 items did show significant differences. The authors were also able to present a result of a factor analysis that was conducted, labeling the three factors "sleep-incompatible behaviors," "sleep-wake cycle behaviors," and "thoughts and attitudes to sleep," which might be a good categorization for sleep hygiene instructions. 
In general, sleep hygiene should be combined with other psychological techniques, such as restriction of bedtime and stimulus control therapy.

Staying awake in bed for too long and extending the bedtime causes a reduction in sleep pressure in the evening, thus leading to a worsening of insomnia symptoms. In this case, restriction of bedtime is a very effective method, though it may sound counterintuitive to a chronic insomnia patient at first glance.

As part of the stimulus control therapy, ${ }^{65}$ all possible interfering objects, such as a clock, a TV set, and a small light, should be moved out of the bedroom and light sources outside the window should be dimmed well by curtains. Digestion and legally available substances (eg, caffeine, nicotine, and alcohol) also interfere with sleep and need to be avoided. In order to be able to fall asleep, the circadian rhythmicity and sleep pressure must be considered. Process " $\mathrm{S}$ " needs to be on a high level, ${ }^{61}$ or in other words, a person needs to be tired, which is more difficult, if they take daytime naps previously. So, therefore, naps should be avoided as long as the patient suffers from insomnia. A list of stimulus control instructions is shown in Table 4 (\# 10 through \#15).

Many insomniacs suffer from ruminating when they are lying in bed and cannot sleep. In this case, typical strategies of a cognitive-behavioral therapy can be applied in this case. Writing down the thoughts a few hours before going to bed or using a "ruminating chair" in the living room for rumination instead of doing it in bed are only the two of common techniques. Unrealistic expectations, eg, about "what and how sleep should be" or the thought of "not being able to slow down" should be modified in therapy in order to resolve these negative sleep-related thoughts and to develop a more relaxed attitude towards sleep.

Psychotherapeutic relaxation techniques are well-tried methods in the treatment of insomnia, especially autogenic training, progressive muscle relaxation, and mindfulness exercises have a positive influence on emotional and cognitive and physiological arousals, which disturb the process of falling asleep. ${ }^{66,67}$ Most evidence exists for the effectiveness of progressive muscle relaxation in the treatment of insomnia. ${ }^{68,69}$ The advantages of progressive muscle relaxation are that the patients can learn it quite quickly, and afterwards, they have an effective method they can use before falling asleep and during longer wake time at night.

The effectiveness of mindfulness meditation for treating insomnia has been examined in several studies. For example, Ong et $\mathrm{al}^{70}$ used a combination of mindfulness exercises and typical cognitive-behavioral therapy methods and found significant improvements in insomnia, as well as a significant correlation between the number of meditation sessions and changes on a trait measure of arousal. Further randomized controlled studies are needed to evaluate the effect of mindfulness meditation on insomnia symptoms, but first results show positive effects.

Gooneratne ${ }^{71}$ presents an overview of first studies concerning complementary and alternative medicine as further treatment options for sleep disturbances, mentioning positive effects of yoga and tai chi, massage therapy, or acupressure in older adults. These forms might have a potential, but they are not well established yet and further research needs to be done.

All these techniques and strategies as a combination are best administered in a group therapy. Trials in the past years have proven persistent efficacy for combination therapy, eg, a 3-year follow-up study done by Backhaus et al. ${ }^{72}$ More details and information on cognitive-behavioral interventions, sleep restriction, stimulus control, or sleep hygiene can be found in the literature of Espie, ${ }^{73}$ Backhaus and Riemann, ${ }^{74}$ Morin, ${ }^{75}$ and Perlis et al. ${ }^{76}$

Due to cost-benefit calculations, practitioners and scientists keep searching for new and less-expansive forms of insomnia therapy. One recent suggestion was the Intense Sleep Retraining treatment, ${ }^{77}$ consisting of sleep deprivation for one night, followed by a series of 50 brief nap opportunities to experience and relearn sleep initiation. In an uncontrolled pilot study, this procedure leads to a reduced sleep onset latency and a decreased nocturnal wake time. Total sleep time increased and was stable for a follow-up period of 2 months. This promising approach should be investigated in future studies, including control conditions and longer follow-up periods.

Morin et a ${ }^{80}$ conducted a study on an internet-based CBT for insomnia, including 22 participants in the intervention group and 23 in a wait-list control group. The intervention included elements of sleep restriction, sleep hygiene, stimulus control, cognitive restructuring, and relapse prevention. Treatment effects were assessed by the Insomnia Severity Index and daily sleep diaries to measure changes in the main sleep variables and insomnia severity. Significant improvements were reported for the Internet group on the Insomnia Severity Index, wake after sleep onset, and sleep efficiency compared with nonsignificant changes in the control group. Depending on the Internet access abilities, this medium seems to be a considerable option for insomnia treatment.

Self-help therapy in the form of nonpharmacological, standardized psychological treatment manuals, which can be 
worked through by the patients themselves, appear to be an inexpensive and accessible alternative for mild to moderate severity insomnia disorders. Yet, van Straten and Cuijpers ${ }^{79}$ in a recent meta-analyses found lowered effect sizes compared to face-to-face therapy interventions in a recent meta-analysis and could not prove any long-term efficacy. However, highquality and large-scaled long-term studies on this topic are missing.

To prove long-term effects of psychological and behavioral treatments, five meta-analyses or systematic reviews have been conducted in recent years. ${ }^{80-84}$ Three of these have focused on studies with elderly people (aged $>55$ or 60 years). A methodological difference to pharmacological studies is the power strengthening opportunity of test-retest analyses, using pre-post change calculations based on within subject variance.

Morin et al included 59 studies to determine the value of pre-post changes on the outcome parameters, such as sleep latency (SL), wake time after sleep onset (WASO), number of awakenings (NOA) and total sleep time (TST), reporting effect sizes $\left(\mathrm{Cohen}^{85}\right)$ of $\mathrm{SL}=0.88$, WASO $=0.65$, $\mathrm{NOA}=0.53$, and TST $=0.42$. Clinical gains were stable after therapy for periods of 6 months in mean. The study included literature from 1974 to 1993, found by "computer search," and focused on the inclusion criteria target problems sleep onset, maintenance or mixed insomnia, nonpharmacological treatment, and group design.

In a similar analysis, Murtagh and Greenwood assessed 66 studies with 1,538 participants, determining the magnitude of pre-post changes compared with control groups. The following effect sizes were calculated: $\mathrm{SL}=0.87, \mathrm{NOA}=0.63$, and TST $=0.49$. In terms of mean differences and percent change, they are reported as follows: SL, $=-24 \min (39.5 \%$ change), $\mathrm{NOA}=-1.2(73 \%$ change $)$, and TST $=+32 \mathrm{~min}$ (9.4\% change). Not mentioning significance values in their analysis, the effect sizes for sleep latency and number of awakening seems to be at least moderate. According to the provided data, the maintenance of clinical gains after discontinuation appears to be given. Murtagh and Greenwood searched the databases PsycLit and MEDLINE, as well as the reference lists of review articles including unpublished studies for the time span of 1973-1993 (inclusion criteria: involvement of a psychological treatment or a combination of psychological treatment, at least five subjects with PI; exclusion criterion: comorbid insomnia).

Pallesen et $\mathrm{al}^{82}$ focused on studies with elderly insomnia patients with a mean age of 60 years and more. Thirteen studies $(n=388)$ were retained for analysis, resulting in data for effect sizes after acute treatment and at follow-up (added in parentheses). The average length of follow-up periods was 6 months. Effect sizes were 0.41 (0.64) for SL, $0.61(0.59)$ for WASO, $0.25(0.66)$ for NOA, and 0.15 (0.37) for TST, leading to the conclusion that behavioral treatments are significant in their efficacy and produce lasting improvements in the elderly. In this analysis, a search in the databases PsycLit and MEDLINE was performed for the years 1966-1998, using the criteria behavioral or psychological treatment, sleep onset, maintenance, or mixed insomnia, and a mean age $>60$ years.

The fourth meta-analysis was conducted by Montgomery and Denis. ${ }^{83}$ It included six studies $(\mathrm{n}=274)$ and its inclusion criteria were RCTs, age older than 60 years, and complaint/ diagnosis of insomnia; exclusion criteria were severe or unstable medical and psychiatric illness, secondary sleep disorder, and failure to meet a checklist of methodological issues for quality purposes. The sources included MEDLINE, EMBASE, CINAHL, PsycInfo, the Cochrane Controlled Trials Register, the National Research Register, and the sleep bibliography of www.webscience.org for a period from 1966 to 2002. The outcome measures included SL, WASO, and TST, as determined by sleep logs. The results showed a minimal reduction of 3 minutes for sleep latency, a moderate reduction in wake time after sleep onset (mean, 22 minutes), and a moderate increase in total sleep time (mean, 14.6 minutes). According to these results, CBT-I seems to be best used for sleep maintenance problems in the elderly.

The last meta-analysis was published by Irwin and colleagues who identified 23 RCTs and evaluated treatment efficacy in elderly adults of more than 55 years of age and middle-aged adults, using the databases Cochrane Controlled Trials Register, PsycInfo, PubMed, Scientific Citation Index (SSCI) for the years 1966-2004. Mean effect sizes of acute treatment were presented for sleep latency $(0.50)$, wake time after sleep onset (0.69), total sleep time (0.17), sleep efficiency (0.74), and subjectively reported sleep quality (0.79). This data confirms previous results indicating that CBT-I is an effective treatment strategy for PI. This study also emphasized the question of whether or not age has a moderating effect on the outcome of behavioral interventions. Taking into account the limitation due to the number of original studies, the improvement in sleep efficiency is given in the elderly cohort, but middle-aged adults did improve to a greater extent. Compared with the control group, older adults did not show any improvement in their total sleep time after behavioral treatment, whereas middle-aged adults did have changes. The results of the criteria, such as sleep 
latency, wakening after sleep onset, and sleep quality, did not show any differences between the age cohorts; so, therefore, behavioral interventions are equally effective across the age groups.

\section{Conclusion and future perspectives}

There is compelling evidence that psychological and pharmacological treatment of insomnia is effective. Hypnotic medications are well evaluated for a short-term usage and, therefore, should only be used for a short period of time in clinical practice, until more long-term studies will be published in the future. The occurrence and management of side effects is an important issue for insomnia patients and must be appreciated in a pharmacological treatment strategy. CBTs are the first-line option for insomnia as long-term effects have been shown conclusively. However, a major problem of CBT is the assessability for most insomnia patients. Internet-based therapy might be a solution for this; in addition, face-to-face treatment could be administered through a stepped-care approach, as recently proposed by Espie. ${ }^{86}$ He developed a hierarchy of delivering CBT on five levels, starting with a self-administered CBT as a basis and manualized, small-group CBT delivered by nurses on the next level, if there is an incomplete therapeutic response, "referring" further upstream, and if increased requirements of expertise, time, and cost are necessary. More experience with this health care model for insomnia patients is necessary to draw further conclusions on availability for a larger proportion of society and cost-effectiveness.

\section{Disclosure}

This was not an industry sponsored work. Dr Riemann has received research support from sanofi-aventis, Omron, Organon, Takeda, and Actelion, participated in speaking engagements for sanofi-aventis, Lundbeck, Servier and GlaxoSmithKline, and served on advisory boards for sanofiaventis, Lundbeck, and GlaxoSmithKline. Dr Spiegelhalder, Ms Hirscher, and Mr Unbehaun have indicated no financial conflicts of interest.

\section{References}

1. American Psychiatric Association. Diagnostic and Statistical Manual of Mental Disorders. 4th ed. Text Revision. Washington, DC: APA; 2000.

2. American Academy of Sleep Medicine. ICSD-2 - International Classification of Sleep Disorders: Diagnostic and Coding Manual, ICSD-2. 2nd ed. Newton, MA: American Academy of Sleep Medicine; 2005.

3. Ohayon MM. Difficulty in resuming sleep or inability to resume sleep and the links to daytime impairement: definition, prevalence and comoridity. J Psychiatr Res. 2009;43(10):934-940.
4. Ohayon MM. Nocturnal awakenings and comorbid disorders in the American general population. J Psychiatr Res. 2008;43(1):48-54.

5. LeBlanc M, Mérette C, Savard J, Ivers H, Baillargeon L, Morin CM. Incidence and risk factors of insomnia in a population-based sample. Sleep. 2009;32(8):1027-1037.

6. National Institutes of Health SotSCS. Manifestations and management of chronic insomnia in adults. Sleep. 2005:1049-1057.

7. Riemann D, Voderholzer U. Primary Insomnia: a risk factor to develop depression? J Affect Disord. 2003;76(1-3):255-259.

8. Neckelmann D, Mykletun A, Dahl AA. Chronic insomnia as a risk factor for developing anxiety and depression. Sleep. 2007;30(7):873-880.

9. Breslau N, Roth T, Rosenthal L. Sleep disturbance and psychiatric disorders: a longitudinal epidemiological study of young adults. Biol Psychiatry. 1996;39(6):411-418.

10. Vgontzas AN, Liao D, Bixler EO, Chrousos GP, Vela-Bueno A. Insomnia with objective short sleep duration is associated with a high risk for hypertension. Sleep. 2009;32(4):491-497.

11. Suka M, Yoshida K, Sugimori H. Persistent insomnia is a predictor of hypertension in Japanese male workers. J Occup Health. 2003; 45(6):344-350.

12. Mander B, Colecchia E, Spiegel K, Kim R, Sannar E, Van Cauter E. Short sleep: a risk factor for insulin resistance and obesity. Diabetes. 2001; 50 Suppl 2:A45.

13. Burgos I, Richter L, Klein T, et al. Increased nocturnal Interleukin-6 excretion in patients with primary insomnia: a pilot study. Brain Behav Immun. 2006;20(3):246-253.

14. Nakajima H, Kaneita Y, Yokoyama E, et al. Association between sleep duration and hemoglobin A1c level. Sleep Med. 2008;9(7):745-752.

15. Ford D, Kamerow D. Epidemiological study of sleep disturbances and psychiatric disorders. JAMA. 1989;262(11):1479-1484.

16. Perlis ML, Giles DE, Mendelson WB, Bootzin RR. Psychophysiological insomnia: the behavioural model and a neurocognitive perspective. J Sleep Res. 1997;6(3):179-188.

17. Riemann D, Spiegelhalder K, Feige B, et al. The hyperarousal model of insomnia: a review of the concept and its evidence. Sleep Med Rev. 2010 Feb;14(1):19-31. Epub 2009 May 28.

18. Bonnet MH, Arand DL. Physiological activation in patients with sleep state misperceptions. Psychosom Med. 1997;59(5):533-540.

19. Freedman RR, Sattler HL. Physiological and psychological factors in sleep-onset insomnia. J Abnorm Psychol. 1982;91(5):380-389.

20. Monroe LJ. Psychological and physiological differences between good and poor sleepers. J Abnorm Psychol. 1967;72(3):255-264.

21. Rodenbeck A, Huether G, Ruether E, Hajak G. Interactions between evening and nocturnal cortisol secretion and sleep parameters in patients with severe chronic primary insomnia. Neurosci Lett. 2002;324(2):159-163.

22. Perlis ML, Merica H, Smith MT, Giles DE. Beta EEG activity and insomnia. Sleep Med Rev. 2001;5(5):363-374.

23. Buysse DJ, Germain A, Hall M, et al. EEG spectral analysis in primary insomnia: NREM period effects and sex differences. Sleep. 2008;31(12):1673-1682.

24. Hauri PJ. Case Studies in Insomnia. New York: Plenum Medical Book Company; 1991.

25. Dauvilliers Y, Morin C, Cervena K, et al. Family studies in insomnia. J Psychosom Res. 2005;58(3):271-278.

26. Zhang B, Wing YK. Sex differences in insomnia: a meta-analysis. Sleep. 2006;29(1):85-93.

27. Kyle SD, Morgan K, Espie CA. Insomnia and health-related quality of life. Sleep Med Rev. 2010;14(1):69-82.

28. Varkevisser M, Van Dongen HP, Van Amsterdam JG, Kerkhof GA. Chronic insomnia and daytime functioning: an ambulatory assessment. Behav Sleep Med. 2007;5(4):279-296.

29. Shekleton JA, Rogers NL, Rajaratnam SM. Searching for the daytime impairments of primary insomnia. Sleep Med Rev. 2010;14(1):47-60.

30. Martin SA, Aikens JE, Chervin RD. Toward cost-effectiveness analysis in the diagnosis and treatment of insomnia. Sleep Med Rev. 2004;8(1):63-72. 
31. Daley M, Morin CM, LeBlanc M, Grégoire JP, Savard J. The economic burden of insomnia: direct and indirect costs for individuals with insomnia syndrome, insomnia symptoms, and good sleepers. Sleep. 2009;32(1):55-64.

32. Kleinman NL, Brook RA, Doan JF, Melkonian AK, Baran RW. Health benefit costs and absenteeism due to insomnia from the employer's perspective: a retrospective, case-control, database study. J Clin Psychiatry. 2009;70(8):1084-1104.

33. Morin CM, Gaulier B, Barry T, Kowatch R. Patient's acceptance of psychological and pharmacological therapies for insomnia. Sleep. 1992;15(4):302-305.

34. Morin CM, Vallières A, Guay B, et al. Cognitive behavioral therapy, singly and combined with medication, for persistent insomnia: a randomized controlled trial. JAMA. 2009;301(19): 2005-2015.

35. Nowell PD, Mazumdar S, Buysse DJ, Dew MA, Reynolds CF III, Kupfer DJ. Benzodiazepines and zolpidem for chronic insomnia: a metaanalysis of treatment efficacy. JAMA. 1997;278(24):2170-2177.

36. Holbrook AM, Crowther R, Lotter A, Cheng C, King D. Meta-analysis of benzodiazepine use in the treatment of insomnia. CMAJ. 2000; 162(2):225-233.

37. Duendar Y, Boland A, Strobl J, et al. Newer hypnotic drugs for the short-term management of insomnia: a systematic review and economic evaluation. Health Technol Assess. 2004;8(24):1-125.

38. Glass J, Lanctôt KL, Herrmann N, Sproule BA, Busto UE. Sedative hypnotics in older people with insomnia: meta-analysis of risks and benefits. BMJ. 2005;331(7526):1169-1175.

39. Buscemi N, Vandermeer B, Friesen C, et al. The efficacy and safety of drug treatments for chronic insomnia in adults: a meta-analysis of RCTs. J Gen Intern Med. 2007;22(9):1335-1350.

40. Krystal AD, Walsh JK, Laska E, et al. Sustained efficacy of eszopiclone over 6 months of nightly treatment: results of a randomized, doubleblind, placebo-controlled study in adults with chronic insomnia. Sleep. 2003;26(7):793-799.

41. Krystal AD, Erman M, Zammit GK, Soubrane C, Roth T. Long-term efficacy and safety of zolpidem extended-release $12.5 \mathrm{mg}$, administered 3 to 7 nights per week for 24 weeks, in patients with chronic primary insomnia: a 6-month, randomized, double-blind, placebo-controlled, parallel-group, multicenter study. Sleep. 2008;31(1):79-90.

42. Ancoli-Israel S, Richardson GS, Mangano RM, Jenkins L, Hall P, Jones WS. Long-term use of sedative hypnotics in older patients with insomnia. Sleep Med. 2005;2:107-113.

43. Erman M, Guiraud A, Joish VN, Lerner D. Zolpidem extended-release $12.5 \mathrm{mg}$ associated with improvements in work performance in a 6-month randomized, placebo-controlled trial. Sleep. 2008;31(10): 1371-1378.

44. Walsh JK. Drugs used to treat insomnia in 2002: Regulatorybased rather than evidence-based medicine. Sleep. 2004;27(8): 1441-1442.

45. Walsh JK, Erman M, Erwin CW, et al. Subjective hypnotic efficacy of trazodone and zolpidem in DMSIII-R primary insomnia. Hum Psychopharmacol. 1998;13(3):191-198.

46. Hajak G, Rodenbeck A, Voderholzer U, et al. Doxepin in the treatment of primary insomnia: a placebo-controlled, double-blind, polysomnographic study. J Clin Psychiatry. 2001;62(6):453-463.

47. Roth T, Rogowski R, Hull S, et al. Efficacy and safety of doxepin $1 \mathrm{mg}$, $3 \mathrm{mg}$, and $6 \mathrm{mg}$ in adults with primary insomnia. Sleep. 2007;30(11): $1555-1561$.

48. Riemann D, Voderholzer U, Cohrs S, et al. Trimipramine in primary insomnia: results of a polysomnographic double-blind controlled study. Pharmacopsychiatry. 2002;35(5):165-174.

49. DeMartinis NA, Winokur A. Effects of psychiatric medications on sleep and sleep disorders. CNS Neurol Disord Drug Targets. 2007; 6(1):17-29.

50. Jeste DV, Blazer D, Casey D, et al. ACNP white paper: update on use of antipsychotic drugs in elderly persons with dementia. Neuropsychopharmacology. 2008;33(5):957-970.
51. Haupt M, Cruz-Jentoft A, Jeste D. Mortality in elderly dementia patients treated with risperidone. J Clin Psychopharmacol. 2006; 26(6):566-570.

52. Buscemi N, Vandermeer B, Hooton N, et al. The efficacy and safety of exogenous melatonin for primary sleep disorders: a meta-analysis. J Gen Intern Med. 2005;20(12):1151-1158.

53. Herxheimer A. Does melatonin help people sleep? BMJ. 2006; 332(7538):373-374.

54. EMEA. Circadin. 2007. Available from: http://www.emea.europa. eu/humandocs/PDFs/EPAR/circadin/H-695-de1.pdf. Accessed on February 10, 2010.

55. Bent S, Padula A, Moore D, Patterson M, Mehling W. Valerian for sleep: a systematic review and meta-analysis. Am J Med. 2006:119(12): 1005-1012.

56. Stevinson C, Ernst E. Valerian for insomnia: a systematic review of randomized clinical trials. Sleep Med. 2000;1(2):91-99.

57. Taibi DM, Landis CA, Petry H, Vitiello MV. A systematic review of valerian as a sleep aid: safe but not effective. Sleep Med Rev. 2007;11(3):209-232.

58. Huang W, Kutner N, Bliwise DL. A systematic review of the effects of acupuncture in treating insomnia. Sleep Med Rev. 2009;13(1): 73-104.

59. Yeung WF, Chung KF, Leung YK, Zhang SP, Law AC. Traditional needle acupuncture treatment for insomnia: a systematic review of randomized controlled trials. Sleep Med. 2009;10(7):694-704.

60. Lee MS, Shin BC, Suen LK, Park TY, Ernst E. Auricular acupuncture for insomnia: a systematic review. Int J Clin Pract. 2008;62(11): 1744-1752.

61. Borbély AA. A two process model of sleep regulation. Hum Neurobiol. 1982;1(3):195-204.

62. Morin CM, Hauri PJ, Espie CA, Spielman AJ, Buysse DJ, Bootzin RR. Nonpharmacologic treatment of chronic insomnia. Sleep. 1999; 22:1134-1156.

63. Stepanski EJ, Wyatt JK. Use of sleep hygiene in the treatment of insomnia. Sleep Med Rev. 2003;7(3):215-225.

64. Adan A, Fabbri M, Natale V, Prat G. Sleep Beliefs Scale (SBS) and circadian typology. J Sleep Res. 2006;15:125-132.

65. Bootzin RR. Stimulus control therapy for insomnia. Proc Am Psychol Assoc. 1972:323-334.

66. Means MK, Lichstein KL, Epperson MT, Johnson CT. Relaxation therapy for insomnia: nighttime and day time effects. Behav Res Ther. 2000;38(7):665-678.

67. Harvey AG, Payne S. The management of unwanted pre-sleep thoughts in insomnia: distraction with imgary vs general distraction. Behav Res Ther. 2002;40(3):267-277.

68. Nicassio PM, Boylan MB, McCabe TG. Progressive relaxation, EMG biofeedback and biofeedback placebo in the treatment of sleep-onset insomnia. Br J Med Psychol. 1982;55(2):159-166.

69. Ebben MR, Spielman AJ. Non-pharmacological treatments for insomnia. J Behav Med. 2009;32:244-254.

70. Ong J, Shapiro S, Manbar R. Combining mindfulness meditation with cognitive-behavior therapy for insomnia: a treatment development study. Behav Ther. 2008;39(2):171-182.

71. Gooneratne NS. Complimentary and alternative medicine for sleep disturbances. Clin Geriatr Med. 2008;24(1):121-138.

72. Backhaus J, Hohagen F, Voderholzer U, Riemann D. Long-term effectiveness of a short-term cognitive-behavioral group treatment for primary insomnia. Eur Arch Psychiatry Clin Neurosci. 2001;1: 35-41.

73. Espie CA. The Psychological Treatment of Insomnia. Chichester, UK: Wiley; 1991.

74. Backhaus J, Riemann D. Schlafstörungen Fortschritte in der Psychotherapie. Goettingen, Bern, Toronto, Seattle: Hogrefe; 1999.

75. Morin CM, Espie CA. Insomnia - A Clinical Guide to Assessment and Therapy. New York: Kluwer Academic/Plenum Publishers; 2003.

76. Perlis M, Jungquist C, Smith MT, Posner D. Cognitive Behavioral Treatment of Insomnia. New York: Springer; 2005. 
77. Harris J, Lack L, Wright H, Gradisar M, Brooks A. Intense Sleep Retraining treatment for chronic primary insomnia: a preliminary investigation. J Sleep Res. 2007;16(3):276-284.

78. Ritterband LM, Thorndike FP, Gonder-Frederick LA, Magee JC, Bailey ET, Saylor DK. Efficacy on an internet-based behavioral intervention for adults with insomnia. Arch Gen Psychiatry. 2009;66(7): 692-698.

79. van Straten A, Cuijpers P. Self-help therapy for insomnia: a metaanalysis. Sleep Med Rev. 2009;13(1):61-71.

80. Morin CM, Culbert JP, Schwartz SM. Non-pharmacological interventions for insomnia: a meta-analysis of treatment efficacy. Am J Psychiatry. 1994;151(8):1172-1180.

81. Murtagh DR, Greenwood KM. Identifying effective psychological treatments for insomnia: a meta-analysis. J Consult Clin Psychol. 1995;63(1):79-89.

82. Pallesen S, Nordhus IH, Kvale G. Non-pharmacological interventions for insomnia in older adults: a meta-analysis of treatment efficacy. Psychother Theory Res Prac Train. 1998;35:472-482.
83. Montgomery P, Dennis J. A systematic review of non-pharmacological therapies for sleep problems in later life. Sleep Med Rev. 2004; $8(1): 47-62$.

84. Irwin MR, Cole JC, Nicassio PM. Comparative meta-analysis of behavioral interventions for insomnia and their efficacy in middleaged adults and in older adults 55+ years of age. Health Psychol. 2006;25(1):3-14.

85. Cohen J. Statistical Power Analysis for the Behavioral Sciences (2nd ed.). Hillsdale, NJ: Lawrence Erlbaum Associates; 1988.

86. Espie CA. Stepped care: a health technology solution for delivering cognitive behavioral therapy as a first line insomnia treatment. Sleep. 2009;32(12):1549-1558.

87. World Health Organization. The ICD-10 Classification of Mental and Behavioural Disorders: Diagnostic Criteria fr Research. Geneva, Switzerland: WHO; 1993.

\section{Publish your work in this journal}

Nature and Science of Sleep is an international, peer-reviewed, open access journal covering all aspects of sleep science and sleep medicine, including the neurophysiology and functions of sleep, the genetics of sleep, sleep and society, biological rhythms, dreaming, sleep disorders and therapy, and strategies to optimize healthy sleep. The journal welcomes

\section{Dovepress}

original research, clinical \& epidemiological studies, reviews \& evaluations, case reports and extended reports. The manuscript management system is completely online and includes a very quick and fair peerreview system, which is all easy to use. Visit http://www.dovepress.com/ testimonials.php to read real quotes from published authors. 